\title{
The Age-Redshift Relation For LRGs
}

\author{
Gaochao Liu ${ }^{1,2}$, Youjun Lu${ }^{1}$, Xuelei Chen ${ }^{1}$, \\ Yongheng Zhao ${ }^{1}$, Wei $\mathrm{Du}^{1}$, and Xianmin Meng ${ }^{1}$ \\ ${ }^{1}$ Key Laboratory of Optical Astronomy, National Astronomical Observatories, Chinese \\ Academy of Science, Beijing 100012, China \\ email: luyj@nao.cas.cn \\ ${ }^{2}$ College of Science, China Three Gorges University, YiChang 443002, China \\ email: gcliu@nao.cas.cn
}

\begin{abstract}
The relative age of galaxies at different redshifts can be used to infer the Hubble parameter and put constraints on cosmological models. We select 23,883 quiescent luminous red galaxies (LRGs) from the SDSS DR7, dividing them into four sub-samples according to their velocity dispersions. Each sub-sample was further divided into 12 redshift bins. The spectra of the LRGs in each redshift and velocity bin were co-added. Adopting the GalexEV/SteLib model, we estimate the mean ages of the LRGs from these combined spectra by the full-spectrum fitting method and find it is consistent with the well known "downsizing" formation of galaxies. Based on the age-redshift relation, we further estimate the Hubble parameter.
\end{abstract}

Keywords. cosmological parameters - cosmology:evolution - galaxies:stellar content

\section{Sample and Results}

We select 23,883 quiescent LRGs from the SDSS DR7. These LRGs are divided into four sub-samples according to their velocity dispersions $\left(\sigma_{v}\right)$ and each LRG sub-sample is further divided into 12 redshift bins. We co-add the spectra of galaxies in each subsample (and each red-shift bin) to obtain combined spectra with significantly higher $S / N(>40)$. These combined LRG spectra are then fitted by a single stellar population by using the GalexEV/SteLib model in the software ULySS, which implements the full spectrum fitting method. The combined spectra, with higher $\mathrm{S} / \mathrm{N}$, enable us to obtain the LRG mean age $\left(t_{\text {age }}\right)$ with considerable accuracy. Our results show that $t_{\text {age }}$ decreases with increasing redshift, and the age of LRGs with higher $\sigma_{v}$ tends to be older than those with lower $\sigma_{v}$, consistent with the well known "downsizing" formation scenario of galaxies. Assuming that the $t_{\text {age }}-\sigma_{v}$ relation follows a power-law, i.e., $t_{\text {age }} \propto \sigma_{v}^{\gamma}$, we find that the mean value of $\gamma$ is $\simeq 0.77$ with a standard deviation of 0.25 .

The age-redshift relation obtained from observations can be directly fitted by $t_{\text {age }}=$ $t_{\mathrm{U}}-t_{\text {form }}$, where $t_{\text {form }}$ is the mean formation time of the quiescent galaxies and assumed to be a constant for each sub-sample, and $t_{\mathrm{U}}$ is the age of the Universe. Assuming a spatially flat $\Lambda$ CDM model, then $t_{\mathrm{U}}=\frac{1}{H_{0}} \int_{z}^{\infty} \frac{d z}{(1+z) \sqrt{\Omega_{\mathrm{m}}(1+z)^{3}+\Omega_{\Lambda}}}$. Using the standard $\chi^{2}$ statistic, we find the best fit (and its uncertainty) for the Hubble constant at the present time is $H_{0}=65_{-3}^{+7} \mathrm{~km} \mathrm{~s}^{-1} \mathrm{Mpc}^{-1}$ if using the age-redshift relation obtained from the LRG sub-sample with the highest $\sigma_{v}$, or $H_{0}=74_{-4}^{+5} \mathrm{~km} \mathrm{~s}^{-1} \mathrm{Mpc}^{-1}$ if using the ageredshift relations obtained from the two LRG sub-samples with high $\sigma_{v}$ simultaneously.

See Liu et al. (arXiv:1208.6502) for more details, 